\title{
EVALUATION OF THE FALSE NEGATIVE RATE IN THE EXAMINATION OF FREEZING OF THE SENTINEL LYMPH NODE IN HBL - RECIFE (PE)
}

Ana Flavia Morais Leda', Darley de Lima Ferreira Filho¹, Nancy Cristina Ferraz de Lucena Ferreira ${ }^{1}$ Hospital Barão de Lucena - Recife (PE) - Brazil.

Introduction: Breast cancer is the most commonly diagnosed cancer in women in the United States and accounts for $26 \%$ of all female cancers. In 2015, approximately 40,290 US women died from breast cancer. In our country, an estimated 59,700 new cases of invasive breast cancer. Then, is very important the early diagnosed of breast cancer and the use of sentinel lymph node probably chance this prognosis of this disease. Objective: To evaluate the false-negative rate of freezing of sentinel lymph node In breast cancer and the factors associated with it. Methods: Twenty-five breast cancer patients who underwent patent blue injection and sentinel lymph node (LS) biopsy between March 2016 and March 2017 in 570 surgery were evaluated for the following variables: age), tumor size, histological characteristics of the tumor (type- and degree of differentiation) and diagnosis of the definitive freezing and histopathological examination of LS. Results: The average age was 56,2, the tumor size was $2,3 \mathrm{~cm}$ and the histhological type was carcinoma ductal invasive in $80 \%$. In a relation the degree of diferenciation, the G2 appear in 19 patients, G1 in 2 cases and G3 in 3 cases. The false-negative rate in the LS freezing test was $8 \%$ ( 2 cases). The specificity was $100 \%$, and sensitivy was $50 \%$. None of the variables evaluated was associated with increase in the incidence of false-negative. In our research, there was no association between tumor size and incidence of axillary metastasis. Conclusion: The false-negative rate of the LS freezing test was $8 \%$ and none of the variables evaluated correlated with this event. No anatamopathological variable studied was associated with the occurrence of false negative 